\title{
RADIOLOGICAL ASSESSMENT OF IONIZING RADIATION IMPACT ON THE TERRESTRIAL NON-HUMAN BIOTA IN LITHUANIA
}

\author{
Marina KONSTANTINOVA ${ }^{a}$, Nina PROKOPČIUKª, Arūnas GUDELIS ${ }^{a}$, Donatas BUTKUS ${ }^{\mathrm{b}}$ \\ ${ }^{a}$ State research institute Center for Physical Sciences and Technology, Institute of Physics, \\ Savanoriu pr. 231, LT-02300 Vilnius, Lithuania \\ ${ }^{b}$ Vilnius Gediminas Technical University, Sauletekio al. 11, LT-10223 Vilnius, Lithuania
}

Submitted 05 Nov. 2014; accepted 17 Apr. 2015

\begin{abstract}
The quantitative assessment of radionuclides transfer to non-human biota using their activity concentration ratios is required for models of predictive doses of ionizing radiation. Based on long-term data regarding activity concentration of radionuclides in the top soil layer of the entire territory of Lithuania, and with the help of ERICA Assessment Tool - a software application that calculates dose rates to selected biota, we estimated the radiological impact on the terrestrial non-human biota with special emphasis on the protected areas located in the vicinity of Ignalina Nuclear Power Plant (INPP). Estimated total dose rates of artificial radionuclides - after-Chernobyl ${ }^{137} \mathrm{Cs}$ and ${ }^{90} \mathrm{Sr}$ as well as discharged by INPP - and natural radionuclides, such as ${ }^{238} \mathrm{U}$ and ${ }^{232} \mathrm{Th}$, were found to be less than ERICA screening value of $10 \mu \mathrm{Gy} \mathrm{h} \mathrm{h}^{-1}$.
\end{abstract}

Keywords: ERICA Tool, terrestrial non-human biota, ${ }^{137} \mathrm{Cs},{ }^{90} \mathrm{Sr},{ }^{238} \mathrm{U},{ }^{232} \mathrm{Th}$, evaluated total dose rate.

\section{Introduction}

Radiological impact on non-human biota in regulatory context is now of interest to a number of international and national bodies and is under scientific investigation. In order to develop predictive models, the quantification of transfer of radionuclides to biota using their activity concentration ratios is highly needed. An ability to predict the radionuclide activity concentrations in biota is an essential component of any approach assessing exposure of non-human biota (Beresford et al. 2008; Beresford et al. 2010; Hosseini et al. 2008; ICRP 2008; Fesenko et al. 2011; Wilson et al. 2010).

Up to the present time, great attention was paid to the human radiation protection in Lithuania: normative acts regulating the human exposure at national levels were validated. However, at present there are no guidelines for non-human biota exposure levels in Lithuania.

Radioactive waste disposal became especially important after the decommissioning of Ignalina Nuclear Power Plant (INPP). The Interim Spent Fuel Storage Facility (ISFSF) is located directly nearby the current INPP facilities. It is important to investigate the present ionizing radiation background in Lithuania. Presented data provide a possibility to put an assessment of the nuclear sites into context and compare the present terrestrial non-human biota exposure due to discharged anthropogenic radionuclides with that of background radiation considering the possible future exposure in the environment by the radioactive waste from the ISFSF.

The aim of this work is to estimate the radiological impact on the terrestrial non-human biota based on longterm data of radionuclide activity concentration in the top soil layer in the whole territory of Lithuania emphasizing the designated area for the ISFSF.

\section{Subject of investigation and methods}

INPP is situated in the north-eastern part of Lithuania close to the borders with Latvia and Belarus on the shore of Lake Druksiai (Fig. 1a). It has two RBMK reactors belonging to the thermal neutron reactor category with an electric capacity of $1500 \mathrm{MW}$. Unit 1 was shut down on 31 December, 2004 and Unit 2 - on 31 December, 2009.

The ISFSF and near-surface repository for redundant materials and waste will be located directly near the current INPP facilities. INPP uses Lake Druksiai as a cooling pond. However, the territory of Lake Druksiai and its

Corresponding author: Marina Konstantinova

E-mail: marina.konstantinova@ftmc.lt 
neighbourhood are protected areas now. These territories are part of the NATURA network (EU Council Directive 1992), a network of protected areas of European Community. Documents related to projects on decommissioning of the INPP were evaluated and agreed (IAEA 2006; Interim storage... 2007). Lake Smalvos is at about $10 \mathrm{~km}$ from the ISFSF while Lake Dysnai is at about $12 \mathrm{~km}$ from the ISFSF, and also are protected areas (Fig. 1b).

There were two most important periods of the Lithuanian territory contamination with ${ }^{90} \mathrm{Sr}$ and ${ }^{137} \mathrm{Cs}$ : the first one covered nuclear weapon tests in the atmosphere in 1945-1980, and the second one started after the Chernobyl Nuclear Power Plant (ChNPP) accident in 1986. Before the Chernobyl accident, data of the ${ }^{137} \mathrm{Cs}$ and ${ }^{90} \mathrm{Sr}$ pollution were derived from measurements taken during 1979-1984 and samples were collected over the whole territory of Lithuania. Data of ${ }^{137} \mathrm{Cs}$ and ${ }^{90} \mathrm{Sr}$ pollution were determined by different research institutes and laboratories in 1987-2000 (Butkus, Konstantinova 2003; Butkus, Konstantinova 2006; Paskauskas, Mazeika 1997). In 1987, the ${ }^{137} \mathrm{Cs}$ distribution over the territory of Lithuania was determined by airborne spectrometry (Butkus et al. 2001).

a)

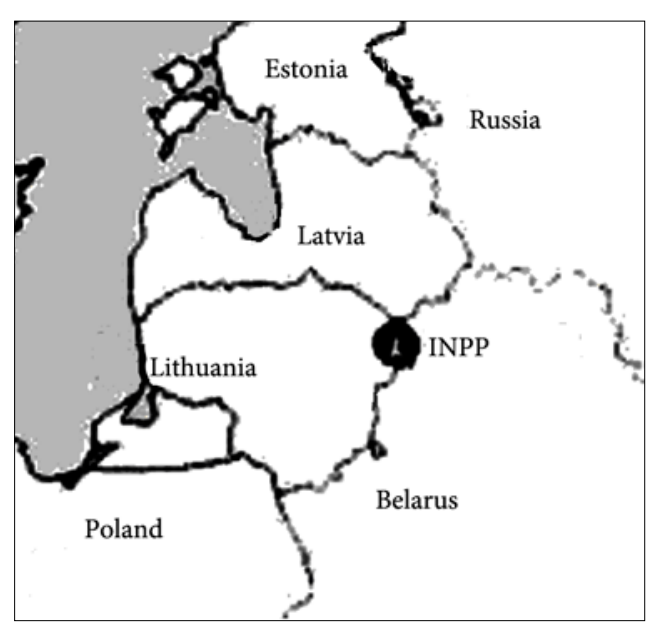

b)

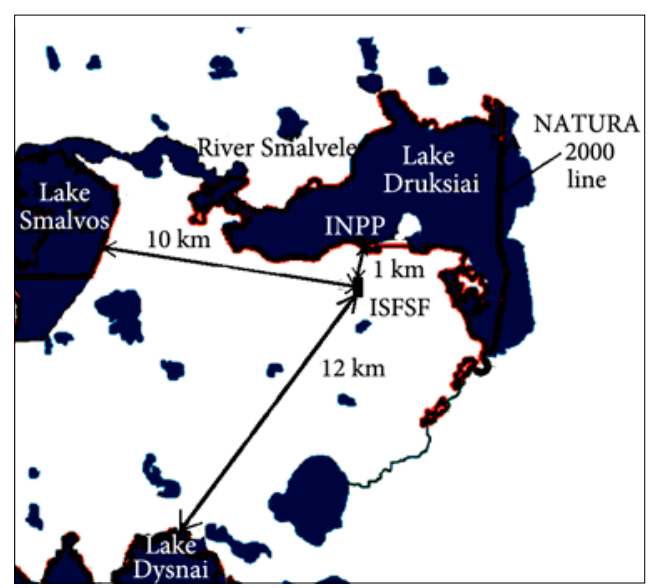

Fig. 1. (a) INPP geographical location, (b) The regional level map with marked INPP, ISFSF locations and outlined NATURA 2000 border (EU Council Directive 1992)
Predominant investigated natural series radionuclides are ${ }^{232}$ Th and ${ }^{238} \mathrm{U}$. Distribution of activity concentration of natural gamma emitters in topsoil was estimated at about the same time over the whole territory of Lithuania. Methods of sampling and measurement were described in (Butkus, Konstantinova 2003).

The major evaluated anthropogenic radionuclides discharged by the INPP are ${ }^{54} \mathrm{Mn},{ }^{60} \mathrm{Co},{ }^{134,137} \mathrm{Cs},{ }^{90} \mathrm{Sr}$ and ${ }^{238} \mathrm{Pu}$. Data on the state of terrestrial ecosystem prior to the operation of the INPP were presented in (Zukauskas et al. 1992). In addition, the results of large-scale radioecological studies had been carried out since 1979 and during the operation of the INPP and they were discussed in (Paskauskas, Mazeika 1997; Butkus et al. 2001; Mazeika 2002; Nedveckaite 2004). These data were then used as input into the model simulations to assess radionuclide transport and the associated radiological doses to nonhuman biota.

Based on these data the exposure dose rates to terrestrial reference organisms were evaluated using ERICA Assessment Tool (Beresford et al. 2008; Howard, Larsson 2008; Brown et al. 2008) version of 2011. ERICA (Environmental Risk from Ionizing Contaminants: Assessment and Management) is a software application that calculates weighted external and internal dose rates to selected biota. Monte-Carlo probabilistic simulation uses distributions of available input data. The result of such stimulation is the probabilistic distribution of the dose rate that facilitates evaluation of the most and the least probable (but possible) distribution values. The value of dose rate not only depends on the reference organism species but also on the dose conversion factor and the ratio of radionuclide concentrations in soil (in our case) and in organisms. Values of the parameters were estimated as indicated in the FASSET (FASSET 2003) database in case of soil.

The databases of ERICA use a number of reference organisms. Each reference organism is representative of a contaminated environment: terrestrial, freshwater or marine ecosystems, and shows the radiological impact on non-human biota. ERICA applies a screening dose rate value of $10 \mu \mathrm{Gy} \mathrm{h}^{-1}$ for all types of organisms. The screening dose rate is a proposed generic (across all species and ecosystems) predicted no effects screening dose rate for application in conservative assessments of screening dose rate. In our work, we investigate terrestrial biota only.

\section{Results and discussions}

In order to compare exposure of terrestrial reference organism due to natural background radionuclides with that of anthropogenic radionuclides the available data on the levels of natural radionuclides were used (Table 1). Special emphasis was placed on measurements of ${ }^{238} \mathrm{U}$ and ${ }^{232} \mathrm{Th}$ top soil activity in previous studies (Butkus, Konstantinova 
2003). ${ }^{238} \mathrm{U}$ and ${ }^{232} \mathrm{Th}$ concentrations in soil were determined by the radiation of their decay products: ${ }^{226} \mathrm{Ra},{ }^{214} \mathrm{~Pb}$, ${ }^{214} \mathrm{Bi}$ for ${ }^{238} \mathrm{U}$ and ${ }^{208} \mathrm{Tl}$ for ${ }^{232} \mathrm{Th}$ (Fig. 2).

Table 1. Examples of estimated total dose rates to terrestrial reference organisms attributed to natural background radionuclides

\begin{tabular}{lcc}
\hline $\begin{array}{c}\text { Terrestrial } \\
\text { reference } \\
\text { organisms }\end{array}$ & $\begin{array}{c}{ }^{238} \mathrm{U} \text { mean total dose } \\
\text { rates, }\left(\mu \mathrm{Gy} \mathrm{h}{ }^{-1}\right)\end{array}$ & $\begin{array}{c}{ }^{232} \text { Th mean total } \\
\text { dose rates, }\left(\mu \mathrm{Gy} \mathrm{h}{ }^{-1}\right)\end{array}$ \\
\hline $\begin{array}{l}\text { Soil invertebrate } \\
\text { Lichen \& }\end{array}$ & $1.44 \cdot 10^{-1}\left(1.70 \cdot 10^{-1}\right)^{*}$ & $4.42 \cdot 10^{-3}\left(7.80 \cdot 10^{-3}\right)$ \\
bryophytes & $3.18 \cdot 10^{-1}\left(3.76 \cdot 10^{-1}\right)$ & $5.17 \cdot 10^{-2}\left(9.13 \cdot 10^{-2}\right)$ \\
Grasses \& herbs & $6.22 \cdot 10^{-2}\left(7.36 \cdot 10^{-2}\right)$ & $2.19 \cdot 10^{-2}\left(3.86 \cdot 10^{-2}\right)$ \\
Shrub & $3.92 \cdot 10^{-2}\left(4.63 \cdot 10^{-2}\right)$ & $8.02 \cdot 10^{-3}\left(1.41 \cdot 10^{-2}\right)$ \\
Tree & $3.98 \cdot 10^{-3}\left(4.70 \cdot 10^{-3}\right)$ & $5.41 \cdot 10^{-4}\left(9.55 \cdot 10^{-4}\right)$ \\
Mammal & $4.22 \cdot 10^{-2}\left(4.99 \cdot 10^{-2}\right)$ & $6.11 \cdot 10^{-5}\left(1.08 \cdot 10^{-4}\right)$ \\
Deer) & $4.83 \cdot 10^{-2}\left(5.71 \cdot 10^{-2}\right)$ & $6.34 \cdot 10^{-5}\left(1.12 \cdot 10^{-4}\right)$ \\
Mammal (Rat) & $6.02 \cdot 10^{-2}\left(7.12 \cdot 10^{-2}\right)$ & $1.95 \cdot 10^{-4}\left(3.44 \cdot 10^{-4}\right)$ \\
Bird & $5.69 \cdot 10^{-2}\left(6.73 \cdot 10^{-2}\right)$ & $1.95 \cdot 10^{-4}\left(3.44 \cdot 10^{-4}\right)$ \\
\hline Amphibians &
\end{tabular}

${ }^{*}$ Note: in brackets $-95^{\text {th }}$ Percentile

The maximum radiation impact on the organism is caused by ${ }^{238} \mathrm{U}$ radiation (Fig. 3), and the total dose rate does not exceed $2.42 \mu \mathrm{Gy} \mathrm{h}^{-1}$. Exposure dose rates of reference organisms reach $0.14 \mu \mathrm{Gy} \mathrm{h}{ }^{-1}$ for soil invertebrates due to the presence of ${ }^{238} \mathrm{U}$ in soil, and $0.05 \mu \mathrm{Gy} \mathrm{h} \mathrm{h}^{-1}$ for lichens and bryophytes due to the presence of ${ }^{232} \mathrm{Th}$ in soil.

a)

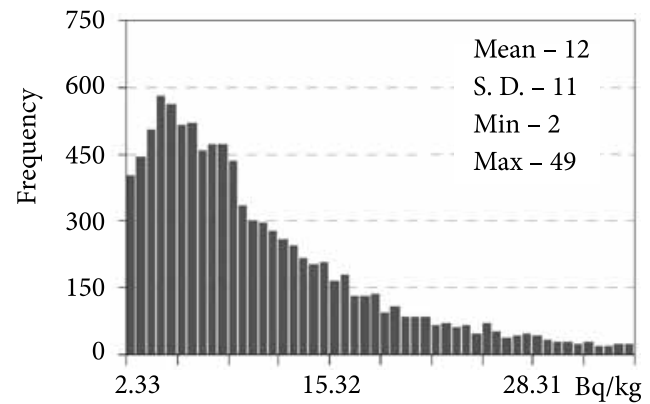

b)

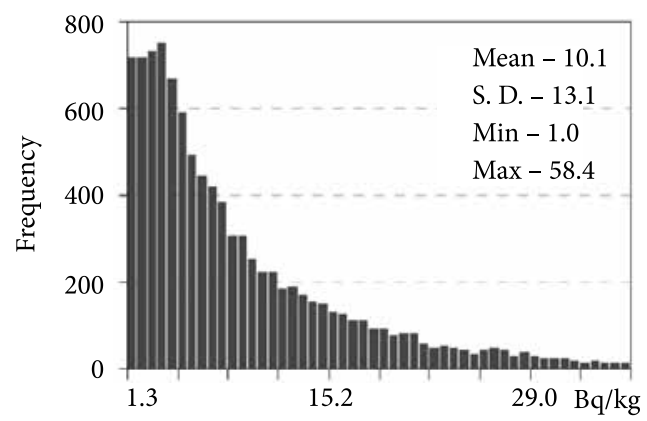

Fig. 2. Frequency histograms of ${ }^{238} \mathrm{U}$ (a) and ${ }^{232} \mathrm{Th}$ (b) activity concentration (d.w.) in Lithuanian soils a)

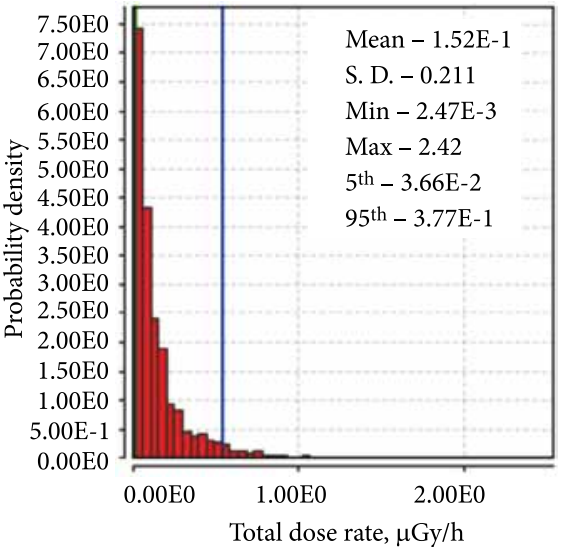

b)

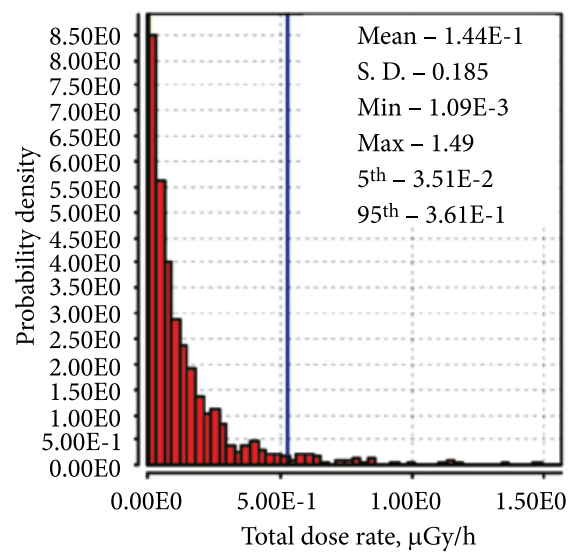

c)

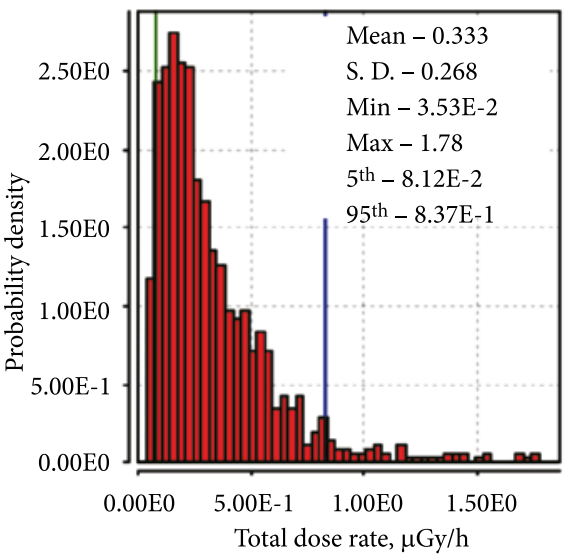

d)

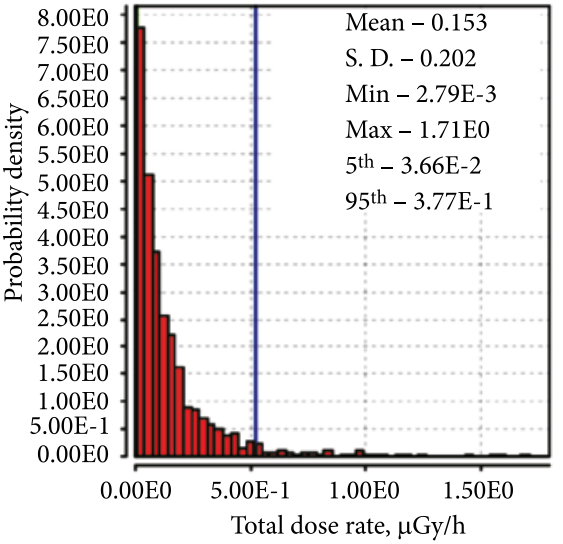

Fig. 3. Examples of estimated total dose rates to terrestrial reference organisms due to ${ }^{238} \mathrm{U}$ only: a) detritivorous invertebrate, b) flying insects, c) lichen \& bryophytes, and d) soil invertebrate (worm) 
After the ChNPP accident Lithuania was directly on the path of contaminated air masses coming from Chernobyl. The regions of elevated level of ${ }^{137} \mathrm{Cs}$ and ${ }^{90} \mathrm{Sr}$ contamination, called "spots", ranging from square meters to square kilometres, were observed (Butkus et al. 1992). At present, contamination with ${ }^{137} \mathrm{Cs}$ and ${ }^{90} \mathrm{Sr}$ is evaluated in the whole territory of Lithuania but the measurements were performed during different time periods. Nowadays ${ }^{137} \mathrm{Cs}$ activity concentration in soil ranges from 6 to $160 \mathrm{~Bq} \mathrm{~kg}^{-1}$ (Fig. 4a). ${ }^{90} \mathrm{Sr}$ activity concentration after the Chernobyl accident nowadays is on average $5 \mathrm{~Bq} \mathrm{~kg}^{-1}$ in Lithuania (Fig. 4b), and includes both the bomb and the Chernobyl fallout.

Figure 5 and Table 2 show the examples of the corresponding ERICA evaluated values of terrestrial ecosystem reference organisms. As it might be seen, all dose rates are far below ERICA $10 \mu \mathrm{Gy} \mathrm{h}^{-1}$ screening value, and reach the maximum value for deer $\left(1.90 \cdot 10^{-2} \mu \mathrm{Gy} \mathrm{h}^{-1}\right.$ on average, and $7.43 \cdot 10^{-2} \mu \mathrm{Gy} \mathrm{h}^{-1}$ at the $95^{\text {th }}$ percentile). Lichen $\&$ bryophytes are also of great concern, dose rate for them is $4.44 \cdot 10^{-2} \mu \mathrm{Gy} \mathrm{h}^{-1}$ at the $95^{\text {th }}$ percentile. The dose rates due to ${ }^{90} \mathrm{Sr}$ are on the order less than that for ${ }^{137} \mathrm{Cs}$ and reach the maximum for reptiles $\left(9.3 \cdot 10^{-2} \mu \mathrm{Gy} \mathrm{h} \mathrm{h}^{-1}\right.$ at the $95^{\text {th }}$ percentile).

The environment of the INPP was investigated in more detail. The artificial radionuclides, originated in the reactors, are found in the local environment. The investigation results of soil activity concentration in the INPP environment are presented in Figure 6, and the data were

a)

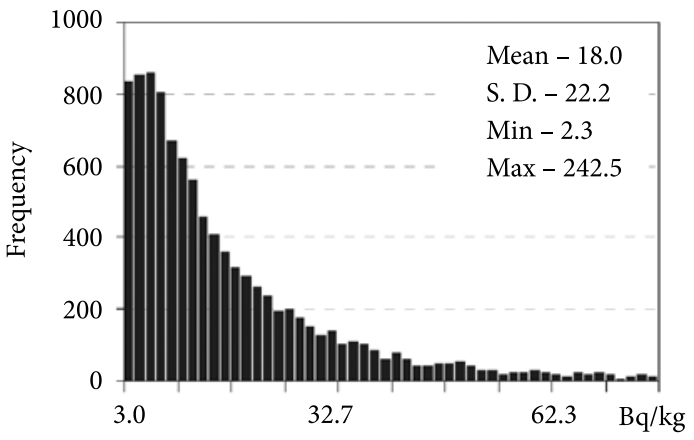

b)

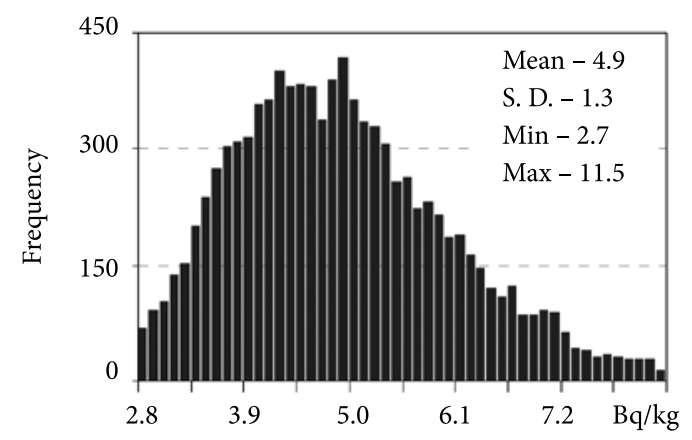

Fig. 4. Frequency histograms of ${ }^{137} \mathrm{Cs}(\mathrm{a})$ and ${ }^{90} \mathrm{Sr}$ (b) activity concentrations after the Chernobyl accident in Lithuanian top soils
Table 2. Estimated total dose rates $\left(\mu \mathrm{Gy} \mathrm{h} \mathrm{h}^{-1}\right)$ to terrestrial reference organisms attributed to artificial radionuclides after the Chernobyl accident

\begin{tabular}{|c|c|c|c|c|}
\hline $\begin{array}{l}\text { Terrestrial } \\
\text { reference } \\
\text { organisms }\end{array}$ & Mean & SD & Min. & Max. \\
\hline \multicolumn{5}{|c|}{${ }^{137} \mathrm{Cs}$} \\
\hline Amphibian & $3.62 \cdot 10^{-3}$ & $4.60 \cdot 10^{-3}$ & $2.79 \cdot 10^{-4}$ & $4.78 \cdot 10^{-2}$ \\
\hline Bird & $4.79 \cdot 10^{-3}$ & $7.26 \cdot 10^{-3}$ & $2.99 \cdot 10^{-4}$ & $6.52 \cdot 10^{-2}$ \\
\hline Bird egg & $2.19 \cdot 10^{-3}$ & $2.25 \cdot 10^{-3}$ & $2.61 \cdot 10^{-4}$ & $2.71 \cdot 10^{-2}$ \\
\hline $\begin{array}{l}\text { Detritivorous } \\
\text { invertebrate }\end{array}$ & $6.23 \cdot 10^{-3}$ & $6.59 \cdot 10^{-3}$ & $7.36 \cdot 10^{-4}$ & $7.27 \cdot 10^{-2}$ \\
\hline Flying insects & $2.43 \cdot 10^{-3}$ & $2.49 \cdot 10^{-3}$ & $2.89 \cdot 10^{-4}$ & $2.70 \cdot 10^{-2}$ \\
\hline Gastropod & $2.41 \cdot 10^{-3}$ & $2.48 \cdot 10^{-3}$ & $2.94 \cdot 10^{-4}$ & $2.78 \cdot 10^{-2}$ \\
\hline $\begin{array}{l}\text { Grasses \& } \\
\text { Herbs }\end{array}$ & $4.06 \cdot 10^{-3}$ & $6.29 \cdot 10^{-3}$ & $2.91 \cdot 10^{-4}$ & $1.04 \cdot 10^{-1}$ \\
\hline $\begin{array}{l}\text { Lichen \& } \\
\text { bryophytes }\end{array}$ & $1.16 \cdot 10^{-3}$ & $1.76 \cdot 10^{-3}$ & $3.34 \cdot 10^{-4}$ & $1.88 \cdot 10^{-1}$ \\
\hline $\begin{array}{l}\text { Mammal } \\
\text { (Deer) }\end{array}$ & $1.90 \cdot 10^{-2}$ & $3.69 \cdot 10^{-3}$ & $3.07 \cdot 10^{-4}$ & $4.02 \cdot 10^{-1}$ \\
\hline Mammal (Rat) & $1.47 \cdot 10^{-3}$ & $2.58 \cdot 10^{-3}$ & $7.87 \cdot 10^{-4}$ & $4.46 \cdot 10^{-1}$ \\
\hline Reptile & $1.34 \cdot 10^{-3}$ & $3.83 \cdot 10^{-3}$ & $3.07 \cdot 10^{-4}$ & $7.16 \cdot 10^{-1}$ \\
\hline Shrub & $1.28 \cdot 10^{-3}$ & $2.30 \cdot 10^{-3}$ & $4.05 \cdot 10^{-4}$ & $4.39 \cdot 10^{-1}$ \\
\hline $\begin{array}{l}\text { Soil } \\
\text { Invertebrate } \\
\text { (worm) }\end{array}$ & $5.97 \cdot 10^{-3}$ & $6.18 \cdot 10^{-3}$ & $7.22 \cdot 10^{-4}$ & $6.68 \cdot 10^{-2}$ \\
\hline Tree & $2.65 \cdot 10^{-3}$ & $3.08 \cdot 10^{-3}$ & $2.32 \cdot 10^{-4}$ & $4.26 \cdot 10^{-2}$ \\
\hline \multicolumn{5}{|c|}{${ }^{90} \mathrm{Sr}$} \\
\hline Amphibian & $2.46 \cdot 10^{-3}$ & $3.74 \cdot 10^{-3}$ & $2.96 \cdot 10^{-5}$ & $5.38 \cdot 10^{-2}$ \\
\hline Bird & $1.79 \cdot 10^{-3}$ & $3.16 \cdot 10^{-3}$ & $2.45 \cdot 10^{-5}$ & $3.76 \cdot 10^{-2}$ \\
\hline Bird egg & $4.09 \cdot 10^{-3}$ & $6.48 \cdot 10^{-3}$ & $3.08 \cdot 10^{-5}$ & $8.46 \cdot 10^{-2}$ \\
\hline $\begin{array}{l}\text { Detritivorous } \\
\text { invertebrate }\end{array}$ & $6.55 \cdot 10^{-4}$ & $1.96 \cdot 10^{-3}$ & $9.46 \cdot 10^{-7}$ & $3.32 \cdot 10^{-2}$ \\
\hline Flying insects & $1.33 \cdot 10^{-4}$ & $1.39 \cdot 10^{-4}$ & $5.31 \cdot 10^{-8}$ & $1.05 \cdot 10^{-3}$ \\
\hline Gastropod & $2.24 \cdot 10^{-4}$ & $9.75 \cdot 10^{-5}$ & $5.88 \cdot 10^{-5}$ & $9.53 \cdot 10^{-4}$ \\
\hline $\begin{array}{l}\text { Grasses \& } \\
\text { Herbs }\end{array}$ & $6.22 \cdot 10^{-4}$ & $4.51 \cdot 10^{-3}$ & $2.84 \cdot 10^{-8}$ & $1.22 \cdot 10^{-1}$ \\
\hline $\begin{array}{l}\text { Lichen \& } \\
\text { bryophytes }\end{array}$ & $1.24 \cdot 10^{-2}$ & $1.22 \cdot 10^{-2}$ & $4.53 \cdot 10^{-4}$ & $1.51 \cdot 10^{-1}$ \\
\hline $\begin{array}{l}\text { Mammal } \\
\text { (Deer) }\end{array}$ & $5.46 \cdot 10^{-3}$ & $6.85 \cdot 10^{-3}$ & $9.79 \cdot 10^{-5}$ & $5.93 \cdot 10^{-2}$ \\
\hline Mammal (Rat) & $5.60 \cdot 10^{-3}$ & $9.44 \cdot 10^{-3}$ & $1.05 \cdot 10^{-4}$ & $1.59 \cdot 10^{-1}$ \\
\hline Reptile & $3.47 \cdot 10^{-2}$ & $6.11 \cdot 10^{-2}$ & $2.47 \cdot 10^{-4}$ & $7.25 \cdot 10^{-1}$ \\
\hline Shrub & $1.27 \cdot 10^{-4}$ & $1.29 \cdot 10^{-4}$ & $4.70 \cdot 10^{-6}$ & $1.18 \cdot 10^{-3}$ \\
\hline $\begin{array}{l}\text { Soil } \\
\text { Invertebrate } \\
\text { (worm) }\end{array}$ & $2.34 \cdot 10^{-5}$ & $2.41 \cdot 10^{-5}$ & $7.14 \cdot 10^{-8}$ & $1.32 \cdot 10^{-4}$ \\
\hline Tree & $1.59 \cdot 10^{-3}$ & $6.21 \cdot 10^{-4}$ & $5.11 \cdot 10^{-4}$ & $4.74 \cdot 10^{-3}$ \\
\hline
\end{tabular}


taken from (Paskauskas, Mazeika 1997; Mazeika 2002). Table 3 shows the examples of corresponding ERICA evaluated exposure values of terrestrial reference organisms at the INPP operation sites. Presented data also are far below ERICA $10 \mu \mathrm{Gy} \mathrm{h}^{-1}$ screening value and reach the maximal value of $0.8 \mu \mathrm{Gy} \mathrm{h}{ }^{-1}$ for deer due to ${ }^{137} \mathrm{Cs}\left(2.37 \mu \mathrm{Gy} \mathrm{h}{ }^{-1}\right.$ at the $95^{\text {th }}$ percentile).

Table 3. Examples of ERICA evaluated exposure values of terrestrial ecosystem reference organisms at INPP operation sites

\begin{tabular}{|c|c|c|c|}
\hline \multirow{2}{*}{$\begin{array}{l}\text { Terrestrial reference } \\
\quad \text { organisms }\end{array}$} & \multicolumn{3}{|c|}{ Mean total dose rates, $\left(\mu \mathrm{Gy} \mathrm{h} \mathrm{h}^{-1}\right)$} \\
\hline & ${ }^{60} \mathrm{Co}$ & ${ }^{134} \mathrm{Cs}$ & ${ }^{137} \mathrm{Cs}$ \\
\hline Soil invertebrate & $\begin{array}{c}5.69 \cdot 10^{-1} \\
(3.64)^{\star}\end{array}$ & $\begin{array}{c}4.46 \cdot 10^{-2} \\
\left(1.82 \cdot 10^{-1}\right)\end{array}$ & $\begin{array}{c}2.54 \cdot 10^{-1} \\
\left(8.25 \cdot 10^{-1}\right)\end{array}$ \\
\hline Grasses \& herbs & $\begin{array}{c}2.10 \cdot 10^{-1} \\
(1.35)\end{array}$ & $\begin{array}{c}2.01 \cdot 10^{-2} \\
\left(8.21 \cdot 10^{-2}\right)\end{array}$ & $\begin{array}{c}1.68 \cdot 10^{-1} \\
\left(5.47 \cdot 10^{-1}\right)\end{array}$ \\
\hline Shrub & $\begin{array}{c}2.21 \cdot 10^{-1} \\
(1.41)\end{array}$ & $\begin{array}{c}3.65 \cdot 10^{-2} \\
\left(1.49 \cdot 10^{-1}\right)\end{array}$ & $\begin{array}{c}5.40 \cdot 10^{-1} \\
(1.76)\end{array}$ \\
\hline Tree & $\begin{array}{c}1.76 \cdot 10^{-1} \\
(1.13)\end{array}$ & $\begin{array}{c}1.83 \cdot 10^{-2} \\
\left(7.46 \cdot 10^{-2}\right)\end{array}$ & $\begin{array}{c}1.15 \cdot 10^{-1} \\
\left(3.75 \cdot 10^{-1}\right)\end{array}$ \\
\hline Mammal (Deer) & $\begin{array}{l}2.24 \cdot 10^{-1} \\
(1.43)\end{array}$ & $\begin{array}{c}1.05 \cdot 10^{-1} \\
\left(4.26 \cdot 10^{-1}\right)\end{array}$ & $\begin{array}{c}8.38 \cdot 10^{-1} \\
(2.73)\end{array}$ \\
\hline \multirow[t]{2}{*}{ Mammal (Rat) } & $\begin{array}{c}5.47 \cdot 10^{-1} \\
(3.5)\end{array}$ & $\begin{array}{c}6.74 \cdot 10^{-2} \\
\left(2.75 \cdot 10^{-1}\right)\end{array}$ & $\begin{array}{c}6.24 \cdot 10^{-1} \\
(2.03)\end{array}$ \\
\hline & ${ }^{90} \mathrm{Sr}$ & ${ }^{54} \mathrm{Mn}$ & ${ }^{238} \mathrm{Pu}$ \\
\hline Soil invertebrate & $\begin{array}{c}1.12 \cdot 10^{-4} \\
\left(2.45 \cdot 10^{-4}\right)\end{array}$ & $\begin{array}{c}6.31 \cdot 10^{-2} \\
\left(2.33 \cdot 10^{-1}\right)\end{array}$ & $\begin{array}{c}5.26 \cdot 10^{-5} \\
\left(9.08 \cdot 10^{-5}\right)\end{array}$ \\
\hline Grasses \& herbs & $\begin{array}{c}2.54 \cdot 10^{-3} \\
\left(5.54 \cdot 10^{-3}\right)\end{array}$ & $\begin{array}{c}2.33 \cdot 10^{-2} \\
\left(8.62 \cdot 10^{-2}\right)\end{array}$ & $\begin{array}{c}2.62 \cdot 10^{-5} \\
\left(4.52 \cdot 10^{-5}\right)\end{array}$ \\
\hline Shrub & $\begin{array}{c}6.08 \cdot 10^{-4} \\
\left(1.33 \cdot 10^{-3}\right)\end{array}$ & $\begin{array}{c}2.52 \cdot 10^{-2} \\
\left(9.33 \cdot 10^{-2}\right)\end{array}$ & $\begin{array}{c}5.72 \cdot 10^{-5} \\
\left(9.87 \cdot 10^{-5}\right)\end{array}$ \\
\hline Tree & $\begin{array}{c}7.64 \cdot 10^{-3} \\
\left(1.67 \cdot 10^{-2}\right) \\
\end{array}$ & $\begin{array}{c}2.01 \cdot 10^{-2} \\
\left(7.45 \cdot 10^{-2}\right)\end{array}$ & $\begin{array}{c}5.72 \cdot 10^{-5} \\
\left(9.87 \cdot 10^{-5}\right)\end{array}$ \\
\hline Mammal (Deer) & $\begin{array}{c}2.72 \cdot 10^{-2} \\
\left(5.94 \cdot 10^{-2}\right)\end{array}$ & $\begin{array}{c}1.21 \cdot 10^{-2} \\
\left(4.49 \cdot 10^{-2}\right) \\
\end{array}$ & $\begin{array}{c}4.25 \cdot 10^{-5} \\
\left(7.33 \cdot 10^{-5}\right)\end{array}$ \\
\hline Mammal (Rat) & $\begin{array}{c}2.59 \cdot 10^{-2} \\
\left(5.66 \cdot 10^{-2}\right)\end{array}$ & $\begin{array}{c}6.02 \cdot 10^{-2} \\
\left(2.23 \cdot 10^{-1}\right)\end{array}$ & $\begin{array}{c}4.25 \cdot 10^{-5} \\
\left(7.33 \cdot 10^{-5}\right)\end{array}$ \\
\hline Soil invertebrate & $\begin{array}{c}1.12 \cdot 10^{-4} \\
\left(2.45 \cdot 10^{-4}\right)\end{array}$ & $\begin{array}{c}6.31 \cdot 10^{-2} \\
\left(2.33 \cdot 10^{-1}\right)\end{array}$ & $\begin{array}{c}5.26 \cdot 10^{-5} \\
\left(9.08 \cdot 10^{-5}\right)\end{array}$ \\
\hline
\end{tabular}

${ }^{*}$ Note: in brackets $-95^{\text {th }}$ Percentile

\section{Conclusions}

Radionuclide activity concentrations in the top soil layer have been used as the basis for a case study in assessing radiological impacts on terrestrial ecosystem non-human biota. The radiation of organisms has the maximum impact due to ${ }^{238} \mathrm{U}$. Exposure dose rates to reference organisms reach $1.70 \cdot 10^{-1} \mu \mathrm{Gy} \mathrm{h}^{-1}$ at the $95^{\text {th }}$ percentile for soil invertebrates due to the presence of ${ }^{238} \mathrm{U}$ in soil, and $9.13 \cdot 10^{-2} \mu \mathrm{Gy} \mathrm{h} \mathrm{h}^{-1}$ at the $95^{\text {th }}$ percentile for lichens and

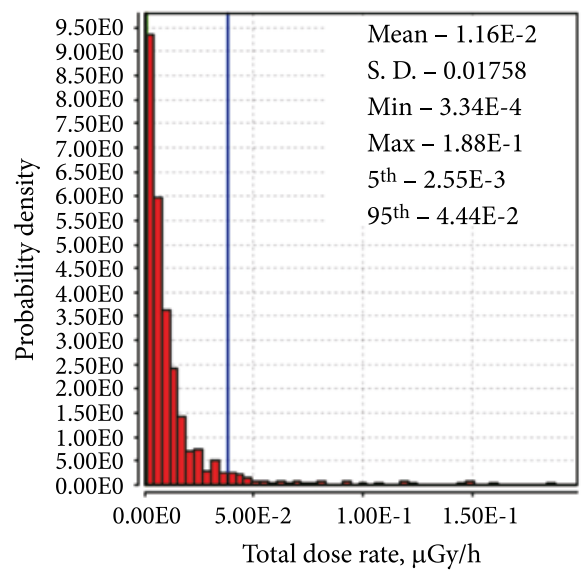

b)

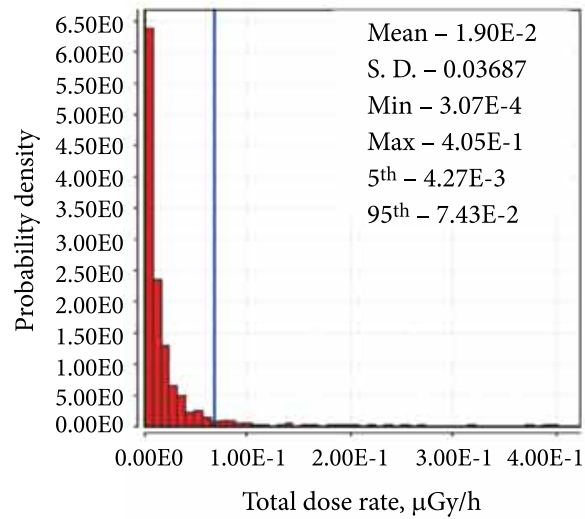

c)

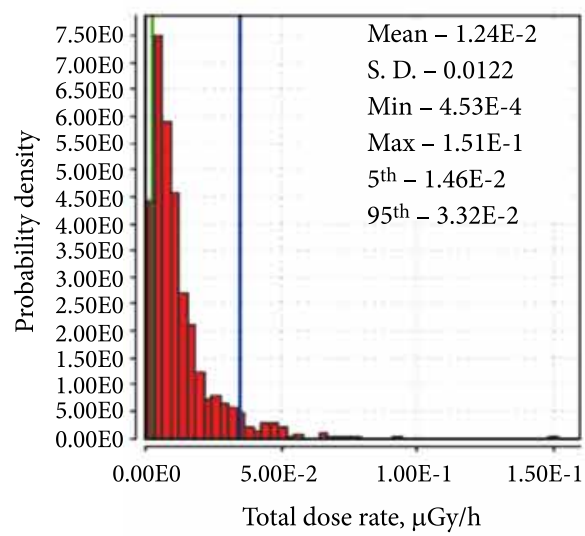

d)

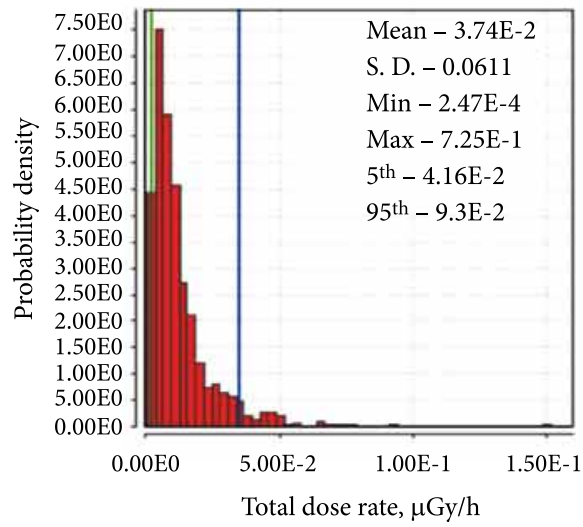

Fig. 5. Examples of estimated total dose rates to terrestrial reference organisms attributed to artificial radionuclides after the Chernobyl accident: lichen \& bryophytes, ${ }^{137} \mathrm{Cs}$ (a); mammal (deer), ${ }^{137} \mathrm{Cs}$ (b); lichen \& bryophytes, ${ }^{90} \mathrm{Sr}$ (c); and reptile ${ }^{90} \mathrm{Sr}$ (d) 
a)

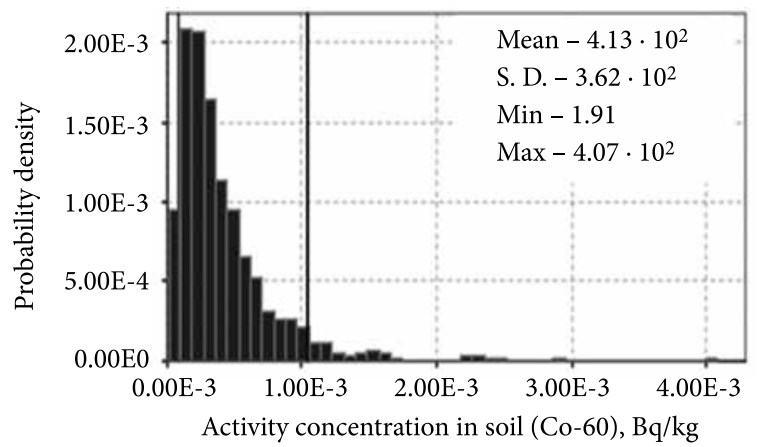

b)

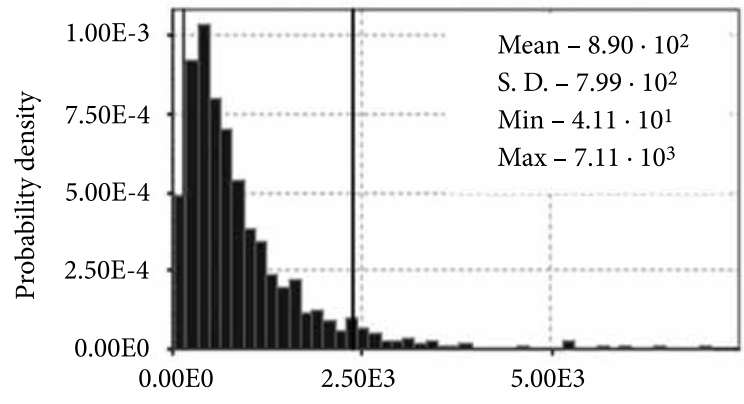

c)

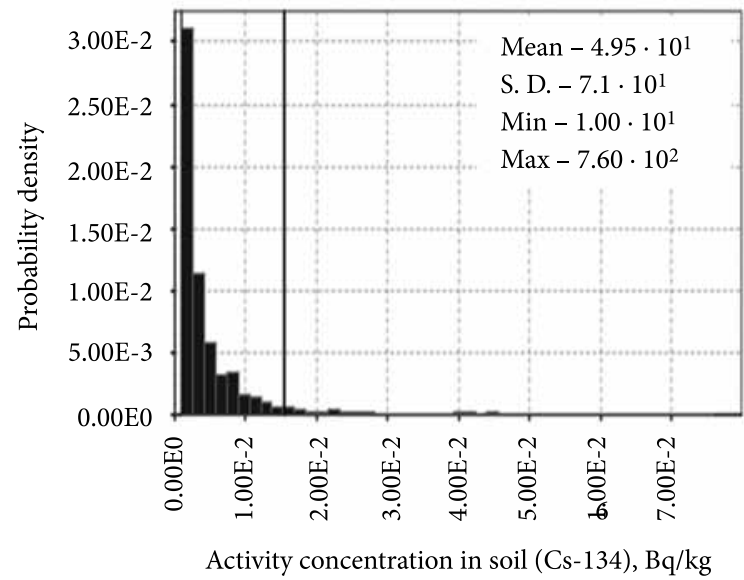

d)

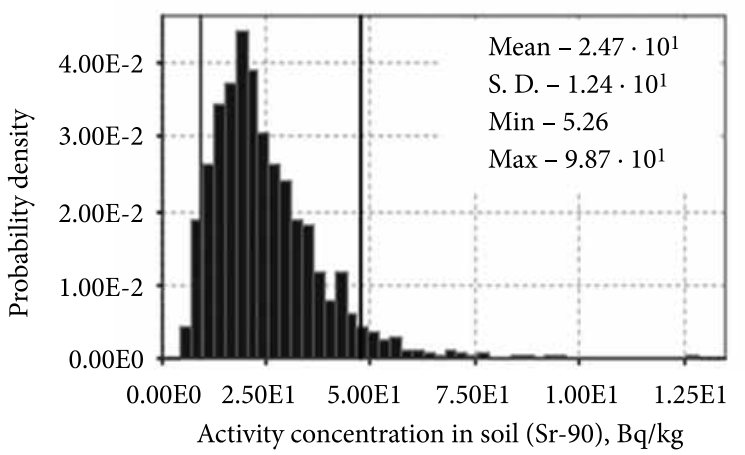

e)

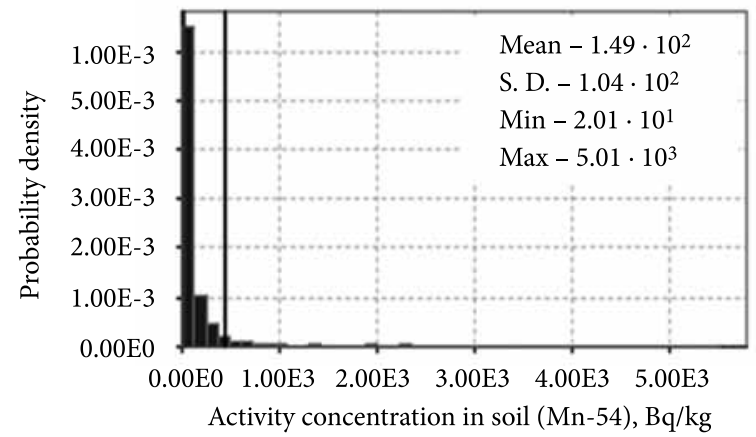

f)

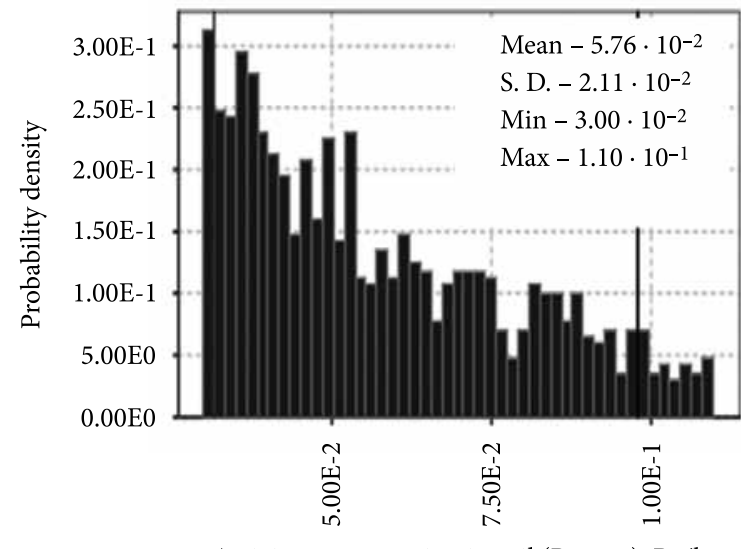

Activity concentration in soil (Pu-238), Bq/kg

Fig. 6. The frequency histograms of anthropogenic radionuclide activity concentration in soils of INPP operation sites

bryophytes due to the presence of ${ }^{232} \mathrm{Th}$ in soil. As for artificial radionuclides, dose rates reach the maximum value for deer $\left(7.43 \cdot 10^{-2} \mu \mathrm{Gy} \mathrm{h} \mathrm{h}^{-1}\right.$ at the $95^{\text {th }}$ percentile). Lichen \& bryophytes dose rate is $4.4410^{-2} \mu \mathrm{Gy} \mathrm{h} \mathrm{h}^{-1}$ at the $95^{\text {th }}$ percentile and dose rates due to ${ }^{90} \mathrm{Sr}$ are on the order less than that for ${ }^{137} \mathrm{Cs}$ and reach the maximum for reptiles $\left(9.3 \cdot 10^{-2} \mu \mathrm{Gy} \mathrm{h} \mathrm{h}^{-1}\right.$ at the $95^{\text {th }}$ percentile). Dose rates due to artificial radionuclide in the vicinity of INPP exceed unity in whole Lithuania, and reach the maximal value of $2.37 \mu \mathrm{Gy} \mathrm{h}{ }^{-1}$ at the $95^{\text {th }}$ percentile for deer due to ${ }^{137} \mathrm{Cs}$.

The evaluated total dose rates due to artificial radionuclides, both after-Chernobyl ${ }^{137} \mathrm{Cs}$ and ${ }^{90} \mathrm{Sr}$, and discharged by INPP, were found to be less than a boundary of $10 \mu \mathrm{Gy} \mathrm{h}{ }^{-1}$, as presented in ERICA approach, therefore, there is no adverse effect on the integrity of non-human biota. The present day total dose rates of up to the $95^{\text {th }}$ percentile for the worst-affected organisms in the regions for nuclear and NATURA 2000 sites are less than $10 \mu \mathrm{Gy} \mathrm{h} \mathrm{h}^{-1}$. Presented data provide a possibility for the comparison of primary non-human biota exposure in context with the environment of radioactive waste and Interim Spent Fuel Storage Facility assumed to be located in this part of Lithuania.

\section{References}

Beresford, N. A.; Barnett, C. L.; Howard, B. J.; Scott, A.; Brown, J. E.; Copplestone, D. 2008. Derivation of transfer parameters for use within the ERICA Tool and the default concentration ratios for terrestrial biota, Journal of Environmental Radioactivity 99: 1393-1407.

http://dx.doi.org/10.1016/j.jenvrad.2008.01.020

Beresford, N. A.; Barnett, C. L.; Brown, J. E.; Cheng, J.-J.; Copplestone, D.; Gaschak, S.; Hosseini, A.; Howard, B. J.; Kamboj, S.; Nedveckaite, T.; Olyslaegers, G.; Smith, J. T. Vives i Batlle, J.; Yu, C. 2010. An international comparison 
of approaches to assess the radiation exposure of wildlife to radioactivity: terrestrial species in the Chernobyl exclusion zone, Journal of Radiological Protection 30: 341-346. http://dx.doi.org/10.1088/0952-4746/30/2/S07

Brown, J. E.; Alfonso, B.; Avila, R.; Beresford, N. A.; Copplestone, D.; Prohl, G.; Ulanovsky, A. 2008. The ERICA Tool, Journal of Environmental Radioactivity 99: 1371-1383. http://dx.doi.org/10.1016/j.jenvrad.2008.01.008

Butkus, D.; Lebedyte, M.; Lubyte, G.; Matusevicius, K.; Mazeika, J. 2001. ${ }^{137} \mathrm{Cs}$ and ${ }^{90} \mathrm{Sr}$ in the soils of Lithuania, Geochemistry International 39: 719-24.

Butkus, D.; Konstantinova, M. 2003. On long-term ${ }^{137}$ Cs migration in the upper layer of Lithuanian soils Lithuanian, Journal of Physics 43: 403-408.

Butkus, D.; Konstantinova, M. 2006. Estimation of capacity of an equivalent dose in the ground air, Vestnik MANEB 11:36-43 (in Russian).

Butkus, D.; Krenevicius, R.; Morkunas, G.; Kandrotas, G.; Korkutis, P. 1992. Investigations of Cs-137 pollution in the territory of Lithuania. Radionuclide pollution in Lithuania and its effects: Workshop papers. Open Society Fund-Lithuania. Vilnius: Academia Press, 21-26.

EU Council Directive 92/43/EEC of 21 May 1992 on the conservation of natural habitats and wild fauna and flora.

FASSET 2003. Deliverable 5. Handbook for assessment of the exposure of biota to ionizing radiation from radionuclides in the environment. Appendix 1: transfer factors and dose coefficient look-up tables. European Commission, Fifth Framework, Contract No. FIGE-CT-2000-00102 [online]. 2003. [cited 12 January 2010]. Available from Internet: https://wiki. ceh.ac.uk/display/rpemain/FASSET+reports

Hosseini, A.; Thørring, H.; Brown, J. E.; Saxe'n, R.; Ilus, E. 2008. Transfer of radionuclides in aquatic ecosystems-default concentration ratios for aquatic biota in the ERICA Tool, Journal of Environmental Radioactivity 99: 1408-29.

Howard, B. J.; Larsson, C.-M. 2008. The ERICA integrated approach and its contribution to protection of the environment from ionizing radiation, Journal of Environmental Radioactivity 99: 1361-1363.

http://dx.doi.org/10.1016/j.jenvrad.2008.04.013

IAEA. 2006. An international peer review of the programme for evaluating sites for near surface disposal of radioactive waste in Lithuania report of the IAEA International Review Team. Vienna.

ICRP. 2008. Environmental protection: the concept and use of reference animals and plants, ICRP Publication 108. Ann ICRP 38: 4-6.

Fesenko, S.; Fesenko, J.; Sanzharova, N.; Karpenko, E.; Titov, I. 2011. Radionuclide transfer to freshwater biota species: review of Russian language studies, Journal of Environmental Radioactivity 102: 8-25.

http://dx.doi.org/10.1016/j.jenvrad.2010.09.006

Interim Storage of RBMK Spent Nuclear Fuel from Ignalina NPP Units 1 and 2 Consortium GNS - NUKEM, LEI - Nuclear Engineering Laboratory. EIA Report. Revision 4. 24 October 2007. LEI Report S/14-658.5.9/EIA-R-04

Mazeika, J. 2002. Radionuclides in geoenvironment of Lithuania. Vilnius: Academia Press.

Nedveckaite, T. 2004. Radiation protection in Lithuania. Vilnius: Kriventa.

Paskauskas, R.; Mazeika, J. (Eds.) 1997. Atomic energy and environment (Lithuanian National scientific programme). The collection of scientific reports. Vilnius: Academia Press.

Wilson, R. C.; Vives i Batlle, J.; Watts, S. J.; McDonald, P.; Jones, S. R.; Craze, A. 2010. An approach for the assessment of risk from chronic radiation to populations of phytoplankton and zooplankton, Radiation Environment Biophysics 49: 87-95.

http://dx.doi.org/10.1007/s00411-009-0254-8

Zukauskas, A.; Jankevicius, K.; Lasinskas, M.; Tamosaitis, J.; Virbickas, J. (Eds). 1992. Thermal power generation and environment (Lithuanian National scientific programme).

Marina KONSTANTINOVA. Dr., State research institute Center for Physical Sciences and Technology, Institute of Physics. Doctor of Science, physics, Institute of Physics, 2002. Publications: co-author of 28 research papers. Research interests: gamma spectrometry, contamination of environment by artificial radionuclides, parameters of 137Cs migration in soil, 137Cs soil-plant transfer, $137 \mathrm{Cs}$ and $3 \mathrm{H}$ release from radioactive waste repository and migration with ground water, 222Rn in atmosphere and soil, human and non-human exposure due to artificial and natural radionuclides, accelerator mass spectrometry, 14C measurements, graphite sample preparation features.

Nina PROKOPČIUK. Dr., State Research Institute Center for Physical Sciences and Technology. Institute of Physics. Doctor of Science, Physics, Institute of Physics, 2013. Co-author of 8 research papers. Research interests: radioecology, radiation protection, atmospheric aerosol particle dynamics and particle optical properties, aerosol particles and human health.

Arūnas GUDELIS. Dr., State research institute Center for Physical Sciences and Technology, Metrology Department, Head of Ionizing Radiation Metrology laboratory. Doctor of Science, Physics, Institute of Physics, 1998. Publications: author and co-author of 64 research papers. Research interests: radionuclide metrology, radionuclides and dosimetry in health, industry and environment, radioactive waste management.

Donatas BUTKUS. Dr Habil, Prof., Dept of Environment Protection, Vilnius Gediminas Technical University (VGTU). Doctor Habil of Science (Environmental Engineering) VGTU, 1999. A corresponding member of International Academy of Ecology and Life Protection. Publications: author of more than 270 research paper, co-author of the monographs "Geophysical problems of atmospheric krypton-85" and "Radionuclides in vegetation". Research interests: accumulation of radioactive noble gases, self-cleaning in the atmosphere, influence of ionizing radiation on geophysical processes, investigation of the consequences of the Chernobyl accident in Lithuania, soil-to-plant transfer of radionuclides. 